\title{
REHABILITATION MANAGEMENT OF STROKE IN YOUNG ADULTS
}

\section{OJOGA Florina ${ }^{122}$,}

\author{
${ }^{1}$ National Institute of Rehabilitation, Physical Medicine and Balneoclimatology, Bucharest \\ 2 "Carol Davila" University of Medicine and Pharmacy
}

\begin{abstract}
Stroke in young adults is particularly a devastating event because it often occurs in healthy-seeming individuals who are in the prime of life and fully involved in family, community and workplace responsibilities. Young adults also have higher expectations of recovery and consequent difficulty in adjusting to disability.
\end{abstract}

Keywords: Stroke, young adults, disability

\section{INTRODUCTION}

Stroke is defined as an acquired injury of the brain caused by occlusion of a blood vessel or inadequate blood supply causing an infarction, or by a hemorrhage within the parenchyma of the brain. $4 \%$ of strokes occur in adults younger than 45 years, being a small fraction of the total events of that sort, but they are an important cause of neurologic impairment in this group.

\section{EPIDEMIOLOGY}

Stroke occurs in young adults more than twice as frequently as spinal cord injury or multiple sclerosis and yet there is limited interest of stroke as a disease affecting young adults. Before 30 years of age, women are more affected because of the risk of pregnancy, childbirth and oral contraceptives, but this incidence reverses with advancing age (1).

\section{ETIOLOGY}

There are many disorders causing stroke in young adults, but they can be grouped in several categories.

Atherosclerotic disease accounts for $20 \%$, cardiac emboli $20 \%$, arteriopathies $10 \%$, coagulopathy $10 \%$ and peripartum cerebrovascular accidents 5\%. Another $20 \%$ can be related to mitral valve prolaps, migraine, oral contraceptives, and $15 \%$ remain unexplained. Drug abuse has been associated with stroke in $4 \%$ to $12 \%$ of cases.

Approximately $75 \%$ of patients younger than 65 years will survive after 5 years or more after the stroke, but the individual survival depends on the cause of stroke and its treatment. The survivors achieve a good functional recovery, but a history of diabetes mellitus, severe deficit on presentation may reduce this thing. The risk for recurrence for those who have suffered a first episode of stroke is about $5 \%$ per year and varies with the presence of risk factors (2). 


\section{SYMPTOMS}

The presenting neurologic symptoms of stroke are the same in young as in elderly patients. The most common of these are emotional effects, pain, muscle stiffness due to spasticity, bladder and sexual dysfunction and fatigue.

\section{EMOTIONAL EFFECTS}

The common emotional effects of stroke are depression, emotional lability and anxiety. Depression occurs in $40 \%$ of patients, its incidence peaks 6 months to 2 years after the incident, and it is frequent in patients with a prior history of alcoholism, or depression or in those with severe stroke.

Depression can be difficult to identify in aphasic patients who cannot respond to questions about mood. They become socially isolated because of language, cognitive and physical deficits. Loss of social interaction and support increases the incidence of depression, being a poor prognostic factor of rehabilitation.

Neurologically mediated emotional lability, also known as emotional incontinence presents with abrupt episodes of crying or laughing and may be a source of distress to the patient and his family. It may complicate the evaluation of the patient's true emotional state.

Patients may experience chronic anxiety after stroke and in some cases there are specific triggers, such as fear of falling while walking with a cane or fear of being left alone.

\section{PAIN}

Pain is a common problem after stroke in young patients. It usually affects the hemiparetic extremities and can be centrally or peripherally mediated.

Shoulder pain is the most frequent, affecting up to $85 \%$ of stroke patients, usually during the first year after stroke.
The potential causes are represented by inferior subluxation of the humeral head, rotator cuff tears, CRPS I (shoulder-hand syndrome), frozen shoulder, impingement syndrome, biceps tendinitis. The careful physical examination and diagnostic tests are useful to confirm the correct diagnostic.

Younger individuals with partially recovery of motor function may develop secondary sprains, tendinitis, nerve palsies in the paretic extremities. The controlateral arm and leg may suffer overuse injuries because of the effort to compensate the weak side.

Heavy use of assistive devices (canes, walkers, braces and splints) may contribute to these injuries and provoke pain.

\section{MUSCLE STIFFNESS DUE TO SPASTICITY}

Stiffness of muscles and joints are frequent symptoms of patients with stroke, due to the evolution of muscle tone from the flaccid to the spastic state that follows during the first several months after the stroke.

Spasticity can be helpful in weight bearing on a leg with little voluntary motor return during gait, but more often complicates the patient's efforts to regain normal motor function.

Spasticity in the upper extremity occurs in a pattern of flexion, adduction, internal rotation and pronation and in the lower extremity the usual pattern is extension with reduced knee flexion, thigh adduction, increased plantar flexion and inversion of the ankle and toe curling. This extensor pattern can be seen during gait and contributes to the slow speed and increased energy cost of hemiparetic gait.

Joint stiffness can be due to contracture, which is defined as shortening of the muscles, ligaments or tendons around a joint, being common in the finger joints of the affected hand or at the shoulder, with contracture of the glenohumeral joint capsule (frozen shoulder). 


\section{BLADDER DYSFUNCTION}

Diminished bladder control with urge incontinence occurs frequently in young stroke patients. Anamnesis should question about chronicity and frequency of the problem, diurnal pattern and presence or absence of needing to void and about abdominal pain and pain during urination.

\section{SEXUAL DYSFUNCTION}

The majority of patients report diminished sexual function after stroke, involving diminished libido, or decreased erectile or ejaculatory function. Decreased libido can correlate with depression that follows the acute episode.

\section{FATIGUE}

Increased fatigue after stroke has been reported in $40-70 \%$ of patients, frequently influenced by coexisting depression. Return to active work and family life can be limited by this symptom.

Usually there is a change in fatigue level after stroke and the daily pattern of fatigue and sleep with insomnia, sleep apnea. Depression and loss of physical conditioning may affect energy levels and also the increased energy cost of hemiparetic gait.

\section{FUNCTIONAL LIMITATIONS IN YOUNG ADULTS WITH STROKE DRIVING}

Return to driving is a necessary step for return to a normal life-style and avoidance of social isolation. There are many negative factors to predict driving performance after stroke: right hemisphere location of stroke, visual-perceptual deficits, reduced attention, impulsivity, poor judgment and lack of organizational skills, correlate with poor performance with driving. Aphasia does not always interfere with driving.

\section{RETURN TO WORK}

The ability to return to work is an important goal for the young patients with stroke and the percent varies from 11 to $81 \%$, due to differing age ranges, definitions of work and disability compensation systems. Factors predictive of success include pure motor or no hemiparesis, good self-care and mobility function, no aphasia or apraxia, advanced education. Poor prognostic factors include cognitive impairment, visual-perceptual impairment, age older than 55 years (3).

The majority of patients return to their previous employer, although young stroke survivors with minimal cognitive impairment may be able to perform easier new jobs.

\section{REHABILITATION TREATMENT}

\section{EMOTIONAL EFFECTS}

Post-stroke depression responds to antidepressant medications, as selective serotonin reuptake inhibitors or tricyclic antidepressants. All the major classes have the potential to lower seizure threshold (4).

The family and community are important resources for the young patient who is struggling with emotional problems to residual disability and altered lifestyle. Management of anxiety should emphasize the less sedating anxiolytics, counseling and environmental manipulation to reduce known triggers.

Neurologic and functional improvement is the best antidote to post-stroke depression. Providing graded and progressive activities gives the patient the chance to improve in parameters of mobility, self-care, language and cognition (5). 
PAIN

Treatment for soft-tissue pain include non-narcotic analgesics and nonsteroidal anti-inflammatory drugs, with specific risks for cardiac, renal, hepatic and gastrointestinal function. Neuropathic and central pain syndromes are treated with gabapentin.

The rehabilitation procedures for soft tissue injuries consist of stretching and strengthening, positioning, electrical stimulation of the affected muscles and heat modalities including hot packs and ultrasound when sensation is preserved to allow their use. TENS and functional electrical stimulation to the supraspinatus and upper trapezius are helpful in shoulder pain as are arm slings that promote optimal glenohumeral alignment.

Subacromial bursa steroid injection are beneficial for patients with post-stroke shoulder pain and botulinum toxin injections provide relief when pain is due to spasticity in specific muscles.

In post-stroke shoulder pain, surgical repair may be considered when rotator cuff tear can be established as the cause and surgical debridement may be required for severe frozen shoulder.

\section{SPASTICITY}

Intercurrent infections, localized sores, stress and anxiety can worsen spasticity and must be treated initially. Tizanidine and gabapentin have analgesic and muscle relaxant actions, so can be used to treat painful spasticity (7).

Mild post-stroke spasticity can often be controlled with a stretching program performed 2-3 times a day and range of motion in a spastic ankle or hand can be preserved with nighttime use of resting splints.

Injection of spastic muscles with botulinum toxin can enhance gait pattern and hand function and reduce pain in young stroke patients (8).
Tendon lengthening and transfers should be considered in young patients when the pattern of spasticity is stabilized. Achilles tendon lengthening may allow improved heel strike in patients with chronic equinovarus due to spastic triceps surae.

\section{BLADDER DYSFUNCTION}

For the stroke patients with urge incontinence due to spastic neurogenic bladder, the anticholinergics oxybutynin and tolterodine are first-line agents for management of detrusor instability. Tricyclic antidepressants provide mild anticholinergic stimulation and can be used to increase bladder capacity.

The rehabilitation treatment for urinary incontinence can be managed with timed voiding every 2 hours during the day, timed fluid intake (none after 9 o"clock p.m.), use of padded clothing or condom catheter and urinal by the bedside.

Pelvic floor strengthening exercises are used for incontinence.

\section{FATIGUE}

The patient must have a normal sleepwake cycle, including maintenance of an appropriate bedtime, avoidance of stimulant beverages late at night and use of hypnotic agents at bedtime, if needed. For the depressed patient with fatigue, a nonsedating antidepressant should be prescribed.

The cardiovascular conditioning program is useful to maximize the patient aerobic capacity and physical status. Patients with physical impairment will benefit from a physical therapist assistance during the adapted conditioning program, using a stationary bicycle, arm ergometer and therapeutic pool (6).

Appropriate bracing and use of assistive devices and gait training can help reduce the energy cost of hemiplegic gait. For the disabled patients, wheelchair propulsion is less fatiguing than walking. 


\section{REFERENCES}

1. Hart R.G., Miller V.T. Cerebral infarction in young adults: a practical approach. Stroke 1983, 14:110-114.

2. Adams H.P., Kappelle LJ. Ischemic stroke in young adults. Arch Neurol 1995, 52:491-495.

3. Naess H, Nyland HY. Long-term outcome of cerebral infarction in young adults. Acta Neurol Scand 2004, 110:107-112.

4. Sacco RL. Risk factors and outcomes for ischemic stroke. Neurology, 1995, 45 (suppl.1): S10-S14.

5. Dennis M, Lewis S. Emotional outcomes after stroke. J Neurol Neurosurg 2000, 68:47-52.

6. Naess $H$, Nyland HL. Fatigue at long-term follow-up in young adults with cerebral infarction. Cerebrovasc Dis 2005, 20:245-250.

7. Gresham GF, Fitzpatrick TE, Wolf PA. Residual disability in survivors of stroke. The Framingham study. N Engl J Med,1975,293:954-956.

8. Black-Schaffer RM, Lemieux L. Vocational outcome after stroke. Top Stroke Rehabil 1994; 1:74-86. 